\title{
Criminologie
}

\section{Prévenir le suicide en prison au Canada}

Les premiers pas d'une politique publique (1970-1987)

Preventing prison suicide in Canada

The first steps of a public policy (1970-1987)

\section{Prevenir el suicidio en prisión en Canadá}

\section{Los primeros pasos de una política pública (1970-1987)}

\section{Claire Guenat et Jean Bérard}

Volume 51, numéro 2, automne 2018

Prise en charge du suicide : entre crime, troubles mentaux et droit de mourir

URI : https://id.erudit.org/iderudit/1054235ar

DOI : https://doi.org/10.7202/1054235ar

Aller au sommaire du numéro

Éditeur(s)

Les Presses de l’Université de Montréal

ISSN

0316-0041 (imprimé)

1492-1367 (numérique)

Découvrir la revue

Citer cet article

Guenat, C. \& Bérard, J. (2018). Prévenir le suicide en prison au Canada : les premiers pas d'une politique publique (1970-1987). Criminologie, 51(2), 61-85. https://doi.org/10.7202/1054235ar
Résumé de l'article

L'article analyse la constitution du suicide carcéral comme un problème public à la fin des années 1970 et au début des années 1980 et rend compte des expertises mobilisées et des registres d'action esquissés par le Service correctionnel du Canada. Il place la genèse de ces réformes dans le cadre plus large des transformations de l'univers carcéral, au croisement entre la promotion des droits des prisonniers, l'accroissement du contrôle des pratiques pénitentiaires, l'ouverture croissante des prisons à des intervenants extérieurs, notamment médicaux, et la reconfiguration du fonctionnement disciplinaire de l'institution. L'article montre que, même s'ils ne sont qu'esquissés dans les années 1980, les lignes de force et les points de tension des politiques de prévention du suicide en prison apparaissent nettement et selon des modalités qui seront ensuite poursuivies et approfondies, notamment autour des enjeux de recueil et partage de l'information, de classification, d'affectation et de surveillance des détenus considérés comme suicidaires. 


\title{
Prévenir le suicide en prison au Canada
}

\section{Les premiers pas d'une politique publique $(1970-1987)$}

\author{
Claire Guenat ${ }^{1}$ \\ Candidate au doctorat \\ École de criminologie, Université de Montréal \\ claire.guenat@umontreal.ca \\ Jean Bérard \\ Maître de conférences \\ École normale supérieure Paris-Saclay \\ Chercheur à l'Institut des sciences sociales du politique (UMR7220) \\ jean.berard@ens-paris-saclay.fr
}

RÉSUMÉ - L'article analyse la constitution du suicide carcéral comme un problème public à la fin des années 1970 et au début des années 1980 et rend compte des expertises mobilisées et des registres d'action esquissés par le Service correctionnel du Canada. Il place la genèse de ces réformes dans le cadre plus large des transformations de l'univers carcéral, au croisement entre la promotion des droits des prisonniers, l'accroissement du contrôle des pratiques pénitentiaires, l'ouverture croissante des prisons à des intervenants extérieurs, notamment médicaux, et la reconfiguration du fonctionnement disciplinaire de l'institution. L'article montre que, même s'ils ne sont qu'esquissés dans les années 1980, les lignes de force et les points de tension des politiques de prévention du suicide en prison apparaissent nettement et selon des modalités qui seront ensuite poursuivies et approfondies, notamment autour des enjeux de recueil et partage de l'information, de classification, d'affectation et de surveillance des détenus considérés comme suicidaires.

MOTS CLÉS - Prévention du suicide, gestion des risques, prison, problème public, Services correctionnels du Canada.

1. École de criminologie, Université de Montréal, Pavillon Lionel-Groulx, C. P. 6128, succ. Centre-ville, Montréal (Québec), Canada, H3C 3J7.

Criminologie, vol. 51, $\mathrm{n}^{\circ} 2$ (2018) 


\section{Introduction}

Le système pénal entretient depuis toujours un lien étroit avec la mort (Foucault, 1975), mais celui-ci est devenu, à l'époque contemporaine, plus problématique et plus ambigu. Du $19^{\mathrm{e}}$ siècle à la seconde moitié du $20^{\text {e }}$ siècle, la mortalité en prison était une réalité peu prise en compte par les politiques pénitentiaires. En France, Jacques-Guy Petit estime que, tout au long du $19^{\mathrm{e}}$ siècle, à peu près 85000 personnes sont mortes dans les maisons centrales, dont probablement plus de 50000 à cause de la surmortalité carcérale (Petit, 1990) ${ }^{2}$. Au Canada, comme l'explique une récente recherche sur les enquêtes menées par les coroners dans des cas de suicide en prison:

même si les préoccupations qui touchent aux suicides et plus globalement aux conditions des détenus existent depuis longtemps, il faudra en fait attendre les années 1970 pour voir se dessiner un véritable changement dans les mentalités concernant les effets nocifs de l'enfermement. (Corriveau, Cauchie et Perreault, 2014)

$\mathrm{Au}$ cours des dernières décennies, cette situation a profondément changé: le suicide a été décriminalisé et est devenu un enjeu de santé publique (Campéon, 2005 ; Cellard, Chapdelaine et Corriveau, 2013). En prison, la prévention du suicide s'est imposée comme un nouveau modèle d'action publique, et est ainsi devenue un axe de réforme important des pratiques carcérales. La création de la Directive du Commissaire numéro 843, intitulée Prévention du suicide et des automutilations, en 1987, marque le début des initiatives correctionnelles en la matière (Service correctionnel du Canada [SCC], 1987). Il ne s'agit alors plus, pour paraphraser, en la déformant, la célèbre formule de Foucault (1976) de «mettre à mort ou laisser mourir», mais d'«empêcher de vivre (libre) et empêcher de mourir» les personnes détenues.

Dans cet article, nous analysons les conditions d'émergence du suicide carcéral comme un problème public et les différents registres d'action esquissés par le Service correctionnel du Canada, des années 1970 aux années 1980. Nous nous intéressons aux acteurs qui ont promu le suicide carcéral comme problème social, à la manière dont ils se sont mobilisés et aux résultats qu'ils ont obtenus. Ces transformations

2. Jacques-Guy Petit (1990) attribue la surmortalité carcérale du $19^{\mathrm{e}}$ siècle aux conditions de détention. Affamés, usés par un travail intensif, peu soignés et assujettis à une discipline intransigeante, les détenus connaissent des taux de mortalité bien supérieurs à ceux de la population libre. 
sont interrogées au prisme des mutations plus larges du fonctionnement des prisons.

En tant qu'objet d'étude sociologique, l'univers carcéral a longtemps été décrit comme un univers total et disciplinaire où l'exclusion, la privation et la désinsertion règnent (Goffman, 1968; Sykes, 1958).

Si certains aspects du projet totalitaire ou disciplinaire perdurent dans les prisons modernes (Mary, Batholeyns et Béghin, 2007; Vacheret, 2006), l'évolution, ces cinquante dernières années, des conditions matérielles et de la gestion de la détention sont le signe d'une transformation partielle de la prison. Une des transformations réside dans son ouverture sur l'extérieur. L'arrivée de nouveaux acteurs, la reconnaissance des droits des détenus, la création d'instances de contrôle externe ou encore la mise en place de nouvelles activités sont autant de symptômes d'un effacement partiel du caractère totalitaire de l'institution (Chantraine, 2000; Rostaing et Touraut, 2016). Ainsi, parmi les nouveaux acteurs apparaissent des médecins, des infirmiers, des psychologues, des enquêteurs correctionnels ou encore des bénévoles susceptibles d'assurer ou de jouer un rôle dans la prévention du suicide en prison.

Cependant, si l'institution du droit pénitentiaire garantit, en théorie, des droits aux détenus, l'existence d'exceptions justifiées par la logique sécuritaire de l'institution carcérale transforme ces droits en privilèges (Bérard, 2014). De plus, si les rapports entre les détenus et les surveillants ont évolué depuis l'analyse de Goffman (1968), ceux-ci se structurent toujours autour d'une mission spécifique: le maintien de l'ordre carcéral. La prison demeure un «dispositif guerrier défensif» où le détenu est l'ennemi de l'intérieur, celui dont il faut se défendre (Chauvenet, Rostaing et Orlic, 2008).

La politique de prévention du suicide carcéral s'inscrit dans ces problématiques. En quoi sa mise en place manifeste-t-elle une responsabilité nouvelle de l'institution carcérale pour la vie des prisonniers et leurs droits, et un contrôle accru de ces actions par des instances autonomes? De quelle manière la prévention du suicide participe-t-elle de l'ouverture des prisons à d'autres acteurs professionnels et à d'autres formes de gestion de l'ordre carcéral? Enfin, comment s'inscrit-elle néanmoins dans le cadre disciplinaire du monde pénitentiaire, ordonné autour du classement et de l'affectation des personnes détenues, de leur surveillance et de leur sanction en cas de comportements interdits? Ce sont les tensions entre ces formes de responsabilisation, d'ouverture et 
de maintien de l'institution carcérale que nous voulons interroger à partir de l'étude de l'émergence du suicide comme enjeu de politique carcérale.

Pour ce faire, nous avons analysé un corpus de sources officielles provenant du SCC, du bureau de l'Enquêteur correctionnel, du ministère du Solliciteur général et du ministère de la Santé et du Bien-être social, d'articles de presse, de travaux universitaires et d'écrits de groupes militants. La sélection de ces documents est issue d'une démarche «en rebond» (Payre, 2009) où chaque trace significative est susceptible de déboucher sur d'autres indices nécessaires à notre compréhension du phénomène étudié. La collecte a visé à l'analyse de sources diverses, à la fois savantes, institutionnelles, médiatiques et militantes, sur une période allant de 1970 à 1987. Les bornes ont été choisies de la manière suivante: d'une part, les premiers travaux et recherches en lien avec la prévention du suicide sur le plan de la communauté scientifique et politique datent des années 1970 (Aujard, 2007; Batt-Moillo, 2005), et d'autre part, 1987 marque l'année à laquelle la première directive du commissaire ${ }^{3}$ relative à la prévention du suicide apparaît. Cette directive ne signifie évidemment pas la fin de la construction de la politique à l'étude: nous la choisissons comme une première borne, car elle indique, selon nous, ce que nous pourrions appeler la fin du début de la problématisation du suicide carcéral, l'achèvement d'un premier moment.

Cette étude demeure pour partie exploratoire, pour deux raisons. L'exploration de la presse a reposé sur un corpus important, mais partiel, dépendant de la disponibilité et de l'accessibilité des sources sur les bases de données mobilisées. De 1970 à 1985, nous avons fondé notre analyse sur le quotidien The Globe and Mail pour l'étendre par la suite à La Presse, au Toronto Star et au Kingston Whig Standard. Par ailleurs, notre exploration des sources militantes est encore limitée: elle porte principalement sur le Québec et nous sommes encore en attente d'autorisations pour l'accès aux archives de l'Office des droits des détenus à la Bibliothèque des archives nationales du Québec de Montréal. Les sources analysées en la matière sont donc celles qui sont d'ores et déjà publiques.

3. Conformément aux articles 97 et 98 de la Loi sur le système correctionnel et la mise en liberté sous condition, le commissaire du SCC peut établir des règles concernant la gestion du Service. Ces règles sont appelées directives du commissaire et font office de politiques correctionnelles. 
Cet article se penche dans un premier temps sur le contexte institutionnel et scientifique ainsi que sur les différents acteurs qui ont contribué à faire du suicide un enjeu de politique publique. Dans un deuxième temps, il analyse l'expertise développée par le SCC, les pistes d'action envisagées et les registres d'action mis en œuvre pour «empêcher les délinquants de se suicider ou se mutiler» (SCC, 1987).

\section{L'émergence d'un enjeu de politique publique}

Un problème est considéré comme public dès lors qu'il est investi par la sphère politique, appelle au débat public ou nécessite l'intervention de l'État (Lascoumes et Le Galès, 2012). La construction d'un problème public représente le processus par lequel un problème est identifié, cadré, justifié, popularisé et mis en politique publique (Neveu, 2015). La première opération consiste à relever les configurations d'acteurs qui œuvrent pour faire reconnaître l'aspect problématique d'une situation. Elle repose sur un ensemble de mobilisations et d'actions collectives (Becker, 1963). La deuxième renvoie aux activités de cadrage, de mise en récit et de problématisation permettant de définir et circonscrire le problème. La troisième fait appel à l'argumentaire des acteurs pour établir la gravité de la situation. La quatrième opération consiste à rendre visible la situation problématique et la cinquième à l'inscrire à l'agenda public. Nous nous penchons sur les trois premières opérations citées ci-dessus: comment comprendre l'émergence de la question du suicide et des automutilations parmi les enjeux de l'action du SCC? Qui s'est mobilisé? Pour quelles raisons? Quelles recompositions sont à l'œuvre dans le cadrage de ce problème? Quels sont les arguments avancés par les «entrepreneurs de problèmes» (Neveu, 2015, p. 41)?

\section{Épidémie de suicide ou attention nouvelle?}

La question du suicide carcéral apparaît comme un enjeu pour le SCC au tournant des années 1980. Plusieurs rapports sont produits pour diagnostiquer le problème et proposer des solutions. Ils débutent par l'explication de l'intérêt des pouvoirs publics pour la question: le SCC a eu le sentiment de faire face à une épidémie de suicide. Un premier rapport de 1979 réalisé par un médecin et une responsable des soins infirmiers, et portant sur les cas de suicide, de tentatives de suicide et d'automutilation dans la région administrative du Québec, explique 
qu'il s'est avéré nécessaire par «ce qui nous semblait une recrudescence» des cas (SCC, 1979a). Un rapport de 1981 expose la même genèse:

En 1979, environ 200 incidents d'automutilation et de suicide ont été signalés dans les pénitenciers canadiens. Ce chiffre représente une augmentation très marquée par rapport à la centaine d'incidents qui avaient été signalés en 1978. Vers la fin de 1979, les comptes mensuels ont révélé que les cas d'automutilation et de suicide continuaient à augmenter. (SCC, 1981a, p. 1)

Pour cette raison, «le Service correctionnel du Canada a donc décidé d'examiner les incidents en vue de formuler des recommandations visant à réduire leur nombre» (SCC, 1981a, p. 1).

Un trait commun de ces rapports est qu'ils commencent par réfuter cette augmentation. Le premier, sur le Québec, constate qu'il y a eu 5 suicides en 1977, contre 10 en 1970 (SCC, 1979a). Surtout, un rapport de 1982 comprend une analyse statistique fouillée et fournit une explication de l'apparente épidémie de suicides: ce sont les instruments de sa mesure qui ont mis le suicide carcéral en crise. En effet, l'installation d'un nouveau système de rapport, d'abord sous-utilisé puis approprié par les agents, a donné le sentiment d'une augmentation, démentie par une recherche directe dans les données des pénitenciers (SCC, 1982).

Cependant, les rapports ne s'arrêtent pas au constat de ce qui pourrait sembler une fausse alerte. C'est ainsi que l'on voit naître l'intérêt spécifique du SCC pour la thématique du suicide. En effet, si les rapports n'indiquent pas d'augmentation, ils désignent la sursuicidité comme un trait spécifique de la population carcérale. La comparaison dans le temps est rapidement évacuée au profit d'une comparaison au même moment entre différentes populations. Le rapport de 1979 rapporte des chiffres de 1977: en population générale masculine, le taux de suicide est de $0,009 \%$, contre $0,17 \%$ en prison (SCC, 1979a). En 1982, un rapport sur le Québec s'inscrit dans la suite de ceux de 1979 et 1981 et établit un diagnostic comparable:

Les taux de décès par suicide pour l'ensemble de la population masculine du Québec sont de 22 pour 100000 et pour les couches d'âge de 20 à 30 ans de 35 pour 100 000. Pour l'ensemble des pénitenciers canadiens, le taux de décès par suicide est de 90 pour 100 000. Le taux de décès par suicide dans les pénitenciers sis au Québec est de 138 pour 100 000. Les suicides dans les pénitenciers au Québec sont de 3 à 4 fois plus élevés que dans la population générale. (SCC, 1982, p. 19) 
Ce qui change durant les années 1970 et 1980 n'est donc pas le suicide en prison lui-même, mais la manière dont le phénomène est inscrit dans un cadre plus large: une intensification de la préoccupation et des mobilisations pour le phénomène suicidaire et la recherche active de populations à risque, parmi lesquelles la population carcérale fait son entrée. Cette entrée s'inscrit, au-delà des murs des prisons, dans un moment d'attention accrue portée au suicide comme un enjeu de santé publique.

\section{Le suicide comme enjeu de santé publique}

En effet, que ce soit au Canada ou à travers le monde, la préoccupation pour le suicide n'est pas nouvelle, mais sa forme change dans les années 1960 et 1970. Les premières associations de lutte contre le suicide voient le jour en 1906 à New York et à Londres et sont suivies de nombreuses initiatives philanthropiques dont le premier centre américain de prévention du suicide à Los Angeles, ouvert en 1958 (Aujard, 2007; Batt-Moillo, 2005). Alors que depuis 1918, prédominait aux États-Unis un courant d'«hygiène mentale» prônant l'assistance aux malades mentaux, 1949 marque l'avènement de l'Institut national de santé mentale qui met l'accent sur la prévention et la promotion du bien-être personnel et collectif. L'approche américaine devient un modèle à suivre pour les chercheurs canadiens et québécois qui, dès 1965 en Ontario et 1979 au Québec, implantent des centres de prévention du suicide (Aujard, 2007).

À l'échelle internationale, l'Organisation mondiale de la santé (OMS) s'implique dans la prévention du suicide dès 1960 en accréditant l'Association internationale pour la prévention du suicide (AIPS). Véritable partenaire de l'Organisation des Nations Unies et de l'OMS, l'AIPS publicise l'importance de la prévention au moyen de conférences internationales. En 1984, le suicide devient un objectif du programme européen de l'OMS de la «Santé pour tous en l'an 2000 » qui, en 1989, recommande à chaque État membre de constituer un programme national de prévention du suicide. Les travaux de ces trois organismes sur la prévention du suicide sont largement diffusés et ont un écho considérable à travers le monde (Aujard, 2007).

Au Canada, la publication d'une étude menée en 1974 par le ministère de la Santé et du Bien-être social provoque une onde de choc au sein du gouvernement et de la population (Leenars, 2000) et participe 
au nouveau cadrage du suicide: peu après la décriminalisation de sa tentative, le suicide devient un problème de santé grave nécessitant une attention importante de la part des pouvoirs publics. Ce cadrage est justifié par plusieurs résultats statistiques qui définissent pour la première fois le suicide comme une cause de décès prématuré chez les Canadiens et comme la deuxième cause de décès chez les Canadiens âgés de moins de 35 ans (Santé et Bien-être social Canada, 1974). Les résultats de cette étude sont repris lors du dixième congrès de l'AIPS, en 1979, durant lequel s'impose la nécessité de constituer une organisation et un lieu d'échange sur le suicide et la manière de le prendre en charge (White, 2003). Cette organisation prend le nom d'Association canadienne de prévention du suicide en 1985. En conséquence, le ministère de la Santé et du Bien-être social décide, en 1980, de créer un groupe d'étude national sur le suicide (Santé et Bien-être social Canada, 1987). C'est dans ce cadre qu'il s'adresse au SCC. Un membre du personnel est nommé pour rejoindre le groupe d'étude national sur le suicide (SCC, 1981b). C'est le début d'un partenariat entre l'instance correctionnelle et le milieu de la santé.

Parallèlement, dès le début des années 1970, le suicide est de plus en plus investi par la sphère scientifique dans un but thérapeutique et préventif. Le développement de l'épidémiologie moderne et la création de la notion de facteur de risque d'une part, et la création de nouvelles méthodes de recherche telles que l'autopsie psychologique d'autre part, contribuent à l'avancée des savoirs étiologiques sur le suicide (BattMoilo, 2005). C'est à cette période, qu'afin de comprendre et déterminer quelles populations sont les plus à risque d'avoir un comportement suicidaire, les premières recherches sur les détenus apparaissent. S'ensuivent de nombreux essais de catégorisation de la population carcérale, proposant de la distinguer par ses profils psychosociaux, pénaux et correctionnels (Beigel et Russel, 1972; Burtch et Erickson, 1979; Topp, 1979).

C'est dans ce contexte à la fois institutionnel et scientifique que l'attention portée par le SCC au suicide prend un sens: elle conduit à appliquer à la population carcérale un cadrage de santé publique et des outils de mesure permettant de cibler les populations à risque.

\section{La prison dans le viseur de la presse}

Un autre élément explique que l'attention du SCC se développe. Dès le début des années 1970, la presse s'empare de la sursuicidité carcérale 
pour dénoncer les ratés de la prison. Les propos de Jean-Pierre Goyer, nouvellement élu Solliciteur général du Canada, sont le point de départ de plusieurs articles dénonçant le nombre élevé de suicides, mais également de décès de manière plus générale en prison. Prenant la parole pour la première fois à la Chambre des communes, Jean-Pierre Goyer se dit «abasourdi» par le nombre de suicides au sein des pénitenciers durant l'année 1970 et qualifie la situation d'alarmante (Goyer is astounded by 19 prison suicides, 1971).

$\mathrm{Au}$ même moment, des articles de presse font des tentatives de dénombrement des suicides en prison. Chaque pénitencier est dès lors placé sous la loupe des journalistes qui tous les ans publient des statistiques et affinent leur analyse du suicide carcéral. Ainsi, en février 1971, on apprend que 23 suicides et 80 tentatives de suicide ont eu lieu depuis septembre 1969, soit 50 fois plus que dans la société libre. En 1972, il y aurait eu 7 suicides contre 11 en 1973 à travers tout le Canada (23 suicides, 80 tries in federal prison since Sept, 1, 1969, 1971; Internal probe can't find reason for 6 suicides at prison this year, 1973). En novembre 1974, le suicide carcéral fait la une du Globe and Mail (Guard carried a knife to cut down persons trying to hang selves, 1974). Les premières lignes reprennent les propos d'un agent de service correctionnel expliquant qu'il a dû faire face à cinq tentatives de suicide par nuit durant la période des fêtes de fin d'année. C'est la raison pour laquelle il garde sur lui en permanence un couteau pour détacher les personnes qui essaient de se pendre.

Le dénombrement des suicides en milieu carcéral s'accompagne d'une volonté de compréhension du phénomène. Le suicide carcéral est alors associé à la surpopulation dans les établissements, au resserrement des conditions d'accès à la libération conditionnelle, à la diminution des permissions de sorties temporaires, à l'ennui, au manque de personnel et au manque de formation du personnel (A jury speaks on suicide in the cells; 1974; Internal probe can't find reason for 6 suicides at prison this year, 1973). La dénonciation de la dureté du régime carcéral s'intensifie avec le temps de même que la remise en question des capacités du SCC à s'occuper de la population à sa charge. La prison est qualifiée de «place of death», de «barbaric» et d'«inbumane» (A jury speaks on suicide in the cells, 1974; Suicides shake public's view of Winnipeg's jail, 1977) et plusieurs articles détaillent des cas de suicide qui auraient, selon eux, pu être empêchés par une intervention des membres du personnel du SCC: «Nurse says rule kept oxygen from banged inmate» en 1974, «Man waited 6 
weeks for mental tests before suicide» en 1976 ou encore, «Jail was warned before suicide» en 1979.

Ce mouvement de dénonciation des échecs de la prison dont l'argumentaire s'appuie sur des données quantitatives et comparatives s'accélère au début des années 1980, avec un nombre croissant de publications et une diversification conséquente du nombre de journaux s'intéressant à la question. Il se transforme au milieu des années 1980 en un appel à trouver des solutions (À quand la fermeture de Parthenais?, 1986; Suicide spurs call for better inmate mental care, 1988). À cette période, la presse et les médias en général prennent une place telle que des chercheurs engagés par le SCC recommandent au sein d'un rapport sur la prévention du suicide d'élaborer un programme qui «inciterait les médias (...) à dédramatiser le suicide d'un détenu et à le traiter avec plus de compassion pour les proches, comme pour les suicides à l'extérieur des pénitenciers» (SCC, 1984).

\section{Le militantisme de l'intérieur et de l'extérieur}

Enfin, une autre forme d'interpellation permet de comprendre l'implication du SCC, celle venue du monde militant. À l'intérieur des prisons, durant les années 1970, se dessine un mouvement de contestation à l'égard des conditions de détention responsables du décès de plusieurs détenus, et surtout, à l'égard du recours à l'isolement. Ce mouvement prend naissance dans le pénitencier de Milhaven, connu à cette époque pour la brutalité de son régime, à la suite du suicide d'Eddie Nolan au sein de l'unité spéciale de détention (Gaucher, 1991). La mobilisation est portée par le groupe Odyssée, un groupe de défense des droits des détenus créé en 1976 par Howie Brown, Leonard Olbey et Chip Tracy, et qui a pour objectif «to bring to the attention of the public what [they] believe to be gross injustices perpetrated by the Canadian Correctional Service» (Smith, 1980). Ils communiquent principalement au moyen de leur bulletin au sein duquel ils partagent des informations et leurs opinions sur l'isolement et ses conséquences, la violence, les décès et le suicide en prison (Gaucher, 1991). En 1978, ils dédient les premières lignes de leur bulletin au «National Prisoner Justice Day», un jour commémoratif instauré un an après le décès d'Eddie Nolan pour se souvenir de ceux qui sont morts en prison (Brown, 1978). À la fin des années 1980, les travaux du groupe Odyssée sortent des murs de la prison grâce à l'implication d'organismes communautaires et de militants. Parmi eux, 
se trouvent notamment Claire Culhane, une célèbre militante engagée dans la défense des droits des personnes détenues et le Conseil des Églises pour la justice et la criminologie (Gaucher, 1991).

Des groupes militants québécois se mobilisent également. On en trouve la première trace dans les actes de colloque publiés par l'AIPS en juin 1979. Lors du $10^{e}$ congrès international sur la prévention du suicide et les interventions en cas de crise, Jean-Claude Bernheim, membre de l'Office des droits des détenus (ODD), prend la parole pour présenter des éléments de compréhension du phénomène de suicide dans les institutions carcérales et lancer un appel aux autorités correctionnelles (AIPS, 1979). Dénonçant, lui aussi, la sursuicidité carcérale, il définit la prison comme un milieu suicidogène et le suicide comme un appel à l'aide. Il critique la dureté des conditions de détention et la vétusté des établissements carcéraux. Il met également l'accent sur le fait qu'un grand nombre de suicides ont lieu dans les ailes d'isolement et invite le SCC à offrir un soutien psychologique aux personnes détenues «pour surmonter le stress inhérent à une telle situation» (AIPS, 1979).

L'intervention de Jean-Claude Bernheim lors du congrès de l'AIPS a un écho auprès du SCC qui publie en août 1979, dans sa revue intitulée Let's Talk, une rubrique reprenant les points clés de sa présentation, mais également ses suggestions pour améliorer la prise en charge des personnes détenues (SCC, 1979b). Dans la foulée, pour répondre à l'appel lancé par l'ODD, le Commissaire du SCC commande aux services correctionnels du Québec une étude faisant le point sur leur situation à l'égard du suicide (SCC, 1979c). L'objectif de ce rapport est de comprendre les raisons pour lesquelles la prévalence des suicides et des incidents d'automutilation est plus importante au sein des pénitenciers québécois que dans les autres provinces. Ce rapport est publié trois ans plus tard sous l'intitulé Étude et recommandations sur les suicides dans les pénitenciers au Québec (SCC, 1982). L'activité de l'ODD se poursuit au courant des années 1980 avec notamment la publication d'un livre visant à lever le voile sur le suicide carcéral «qui n'a jamais suscité de compassion de la part des autorités carcérales» et à «humaniser» la prison (Bernheim, 1987, p. 316).

Laction d'un ensemble d'acteurs divers explique donc l'attention portée par le SCC à la question du suicide au tournant des années 1970 et 1980. Ce n'est pas tant la pratique en elle-même qui apparaît ou augmente, que la mobilisation d'entrepreneurs de problèmes issus à la 
fois du monde de la santé publique à l'échelle nationale et internationale, du monde militant mobilisé sur les questions carcérales et du monde médiatique. Ces acteurs divers tissent des interrelations qui constituent progressivement un réseau mobilisé partageant des constats alarmants, l'analyse de leurs causes et des propositions. Ce réseau produit un cadrage du suicide carcéral comme enjeu de santé publique concernant une population à risque et prenant place au sein d'une institution disciplinaire de plus en plus sommée de s'ouvrir et de rendre des comptes à des acteurs externes, officiels ou non, jouant un rôle de contrôle, officiel ou non.

\section{Construction d'une expertise et des premières pistes d'action}

L'attention de l'administration s'est dès lors portée sur l'affinement de l'analyse de ces comportements. Il s'est agi pour elle de développer des savoirs experts, c'est-à-dire des savoirs désignant des causes, articulés à des formes d'action possible sur ces causes. C'est dans ces tentatives que se dessinent les registres d'action envisagés par le SCC et que commence à se révéler leur tension avec le fonctionnement disciplinaire des prisons.

La constitution d'une expertise entre objectivation statistique et tentatives de compréhension

Les textes écrits à la fin des années 1970 et au début des années 1980 portent la trace du changement de regard sur le suicide en détention. Le rapport remis en 1979 par un médecin et une responsable des soins infirmiers est le dernier signe d'une époque en train de finir. Il constate la sursuicidité carcérale, mais il la renvoie à deux causes. La première relève selon lui d'une sur-déclaration: les suicides en prison seraient tous enregistrés alors qu'une partie d'entre eux sont cachés dans la population générale. Par ailleurs, les auteurs estiment que la «qualité de population» des détenus explique la fréquence des suicides: ceux-ci sont ainsi qualifiés comme «immatures», «caractériels», «polytoxicomanes» ou ne peuvent supporter la perspective de leur procès ou d'un règlement de compte s'ils sont jugés comme des délateurs par leur milieu professionnel. 


\section{F I G U RE 1}

Un cas de suicide vu par le corps médical au Québec dans un rapport de 1979 (SCC, 1979a)

\section{ETUDE DES CAS INDIVIDUELLEMENT}

INSTITUTION LAVAL

\section{Serge Desgagnes $(32$ ans)}

Suicide par pendais on le 9 mai 1979 vers 00:05 hre au Bloc No. 1 .

Ce dêtenu achevait sa sentence et il êprouvait beaucoup de difficultes avec les autres détenus. C'Etait un délateur dont l'avenir était sérieusement compromis autant à l'interrieur qu'd l'exterieur du pénitencier.

Il êtait previsible qu'il se passerait quelque chose. Une première tentative de perdaison avait en lieu au Leclerc en aoat 78 . Tout ce qui pouvait Etre fait pour lui par le secteur médical l'a êté: visites du médecir de l'institution, consultations en psychiatrie en 78 et 79 , protection de l'hôpital à plusieurs reprises.

Il s'est suicide pour une affaire propre au milieu criminel. Il n'avait qu'une seufe issue; il a préféré se donier la mort plutôt que de se jacire tuer à l'intêrieur ou à l'extêrieur.

Enfin, le rapport comprend les automutilations et les menaces de se suicider comme des manières d'obtenir des faveurs dans un milieu contraint: par exemple, un détenu qui voudrait «purger sa sentence à [l'institut Pinel]» se lacère pour y être envoyé, après avoir fait une prise d'otage dans le même but et avoir été renvoyé au pénitencier. Ce qui domine ces explications est l'idée que les prisonniers suicidés ont préféré «la solution la plus facile» (SCC, 1979a).

Pour cette raison, le rapport estime que rien n'aurait pu être fait pour empêcher les passages à l'acte. Il adopte une lecture des actes suicidaires et des automutilations que l'on peut qualifier de fatalisme moraliste. Le texte forme une charnière: il manifeste l'attention nouvelle portée au geste suicidaire en détention. Même si la réponse consiste à montrer que rien n'aurait pu être fait, il a été écrit comme une réponse à une interpellation qui a fait de ces pratiques un enjeu pour le SCC. Pour autant, il porte fortement la marque de la déconsidération morale portée sur les auteurs de gestes suicidaires, héritage de leur longue criminalisation (Cellard et al., 2013; Godineau, 2002).

C'est contre ce cadrage moral et fataliste que vont se constituer, les années suivantes, des formes nouvelles d'expertise. On en trouve la trace notamment dans les rapports remis aux SCC en 1981(a) et 1982. La rupture repose sur deux autres présentations savantes des gestes suicidaires.

La première vise à spécifier les fractions de la population carcérale qui présentent un plus grand risque suicidaire (SCC, 1981a). Le rapport de 1981 opère de nombreuses analyses statistiques pour déterminer des 
personnes et des moments à risque. Il conclut par exemple, sur les «automutilations non fatales», que leur nombre est stable dans le temps, qu'elles sont plus fréquentes dans les prisons de haute sécurité, en isolement et en unité spéciale de détention, mais inexistantes dans les prisons de sécurité minimale. Il montre également que la plupart des cas ont lieu dans les trois mois après l'arrivée dans un établissement, et que les femmes présentent plus de risque - mais les données sont, sur ce point, trop faibles pour conclure. Pour les cas de suicide, ils sont également plus importants dans les établissements à sécurité maximale, et à l'isolement, un peu plus important chez les personnes les moins diplômées et les auteurs de crime contre les personnes. Cette production de savoirs individualisés sur les risques suicidaires que représente chaque membre d'une population carcérale traduit l'installation précoce d'un nouveau modèle de gestion des risques en milieu carcéral (Chantraine, 2006).

Ces nombreux tableaux manifestent cependant l'échec relatif de la caractérisation d'une population à risque: les caractéristiques des individus sont légèrement corrélées avec le risque de suicide, mais ce n'est pas très probant. Ce qui l'est davantage, et s'inscrit dans une ligne d'analyse qui sera par la suite largement répétée, est la caractérisation du lien entre les suicides et tentatives de suicide, les moments (l'arrivée, par exemple) et le niveau de sécurité de la détention (sécurité maximale, unité spéciale et isolement).

F I G URE 2

Une approche statistique pour déterminer des risques de passage à l'acte, dans un rapport de 1981 (SCC, 1981a)

$\frac{\frac{\text { Classement recoupe des incidents d'automutilation }}{\text { selon l'endroit et selon que l'incident a été ou non }}}{\frac{\text { classé comme une tentative de suicide }}{\text { Tout le Canada } 1979}}$

\begin{tabular}{|c|c|c|c|c|c|c|}
\hline & \multicolumn{2}{|c|}{$\begin{array}{l}\text { Tentative de } \\
\text { suicide }\end{array}$} & \multicolumn{2}{|c|}{$\begin{array}{c}\text { Pas une tentative } \\
\text { de suicide }\end{array}$} & \multicolumn{2}{|c|}{ Total } \\
\hline & Nbre & $\frac{9}{8}$ & Nbre & $\frac{3}{8}$ & Nore & 8 \\
\hline Ségrégation & 13 & 24 & 40 & 76 & 53 & 100 \\
\hline $\begin{array}{l}\text { Unités spéciales } \\
\text { de détention }\end{array}$ & 3 & 43 & 4 & 57 & 7 & 100 \\
\hline Isolement protecteur & 3 & 6 & 32 & 94 & 35 & 100 \\
\hline $\begin{array}{l}\text { Cellules ordinaires } \\
\text { et ailleurs }\end{array}$ & 24 & 18 & 111 & 82 & 135 & 100 \\
\hline TOTAL & 43 & 19 & 187 & 81 & 230 & 100 \\
\hline
\end{tabular}

Il est facile de voir que les tentatives de suicide ont représenté un plus grand pourcentage du nombre total des incidents dans les unités de ségrégation et les unités spéciales de detention que dans les cellules ordinaires. Par contre, la fréquence relative des tentatives de suicide dans les cellules d'isolement protecteur est assez faible. 
La seconde forme d'expertise repose sur une approche qualitative du parcours des personnes qui se sont donné la mort. L'analyse porte alors sur les gestes suicidaires dont «l'histoire... même si elle est individuelle, doit être comparée et associée à l'histoire d'autres suicides survenant à une même époque dans une aire géographique définie» (SCC, 1982). Une telle approche s'inscrit explicitement dans la ligne définie par le ministère des Affaires sociales du Québec en mars 1982 (Comité de santé mentale du Québec, 1982). Le rapport de 1982 confirme la distinction du risque selon le niveau de sécurité et concentre son attention sur le bloc cellulaire $\mathrm{n}^{\circ} 1 \mathrm{du}$ pénitencier Laval, dans lequel l'administration concentre tous les cas à problème, et qui offre des conditions matérielles qualifiées de «lamentables» (SCC, 1982).

Plus largement, l'objectif général du rapport est de rompre avec le fatalisme du texte de 1979, auquel il s'oppose explicitement. Pour ce faire, en rassemblant les traits des histoires de suicide, il dresse un portrait-robot $\mathrm{du}$ geste suicidaire:

Dans les pénitenciers, un suicide est un geste posé à la suite d'une situation de crise par un détenu de 20 à 30 ans. Les tensions liées à l'incarcération sont amplifiées s'il est classé parmi des détenus plus agressifs et violents que lui et placé dans des conditions de détention où ses besoins spécifiques en termes de programme de réhabilitation ne sont pas remplis. Il a fait un séjour en protection au bloc cellulaire $\mathrm{n}^{\circ} 1$. Ce détenu a avisé au moins une personne dans le pénitencier de son état de détresse et de son intention de mettre fin à ses jours. Il a déjà fait plusieurs tentatives. Il a échangé régulièrement avec son agent de gestion de cas qui parfois n'arrivait pas à comprendre l'intensité de ses difficultés et se sentait trop seul pour y faire face. Le psychologue l'a évalué à fond. Les psychiatres l'ont rencontré et déclaré qu'il n'était ni psychotique ni névrotique et l'ont parfois admis à l'Institut Pinel. Les services médicaux ont pansé ses blessures et signalé son cas aux administrateurs. Les agents de sécurité selon leur quart de travail ont observé ses crises et ont informé leurs supérieurs. (SCC, 1982)

Le point central de la démonstration porte sur les signes avant-coureurs: contrairement à l'idée d'un acte imprévisible, le rapport met l'accent sur les différentes interactions avec des membres du personnel au cours desquelles l'intention suicidaire a été formulée. Le couplage de l'analyse statistique des populations et de l'analyse des parcours ouvre la possibilité d'actions ciblées à des moments et sur des personnes spécifiques, écartant le double spectre de la totale incertitude sur la survenue de ces gestes et de l'impossibilité de dissuader les personnes qui sont décidées à y recourir. L'enjeu est alors de concevoir des formes nouvelles d'action possibles. 


\section{L'esquisse de répertoires d'action}

Les rapports du SCC montrent qu'au tournant des années 1980, peu d'actions du SCC sont explicitement consacrées à la prévention du suicide (SCC, 1981a, 1982). La plupart des procédures inscrites dans les directives du commissaire, les instructions divisionnaires ou les ordres permanents se concentrent principalement sur l'état de santé, les habiletés de socialisation et le risque que représentent les détenus en matière de sécurité.

Le travail essentiel de ces premiers rapports est de passer de l'analyse des causes à des propositions d'action dans une optique de normalisation des pratiques. Trois grands répertoires d'action sont ainsi abordés.

Le premier est relatif à la circulation de l'information, notamment au moment de l'arrivée des détenus transférés. Les rapports mettent l'accent sur l'absence de formalisation des circuits de communication, qui rend la transmission des informations relativement aléatoire et dépendante de la bonne volonté des personnels concernés. Le rapport de 1981(a) révèle que «la plupart des mesures et procédures d'identification consistent en des communications officieuses entre les membres du personnel» (p. 13). Pour cette raison, les premières pistes d'amélioration proposées portent sur la transmission des dossiers, ainsi que sur le repérage rapide des risques suicidaires à l'entrée dans un établissement. Le même rapport recommande ainsi la mise en place d'une procédure d'identification à l'arrivée des «détenus susceptibles de s'infliger des blessures ou de tenter de se suicider» (p. 11). Il recommande également la mise en place d'un programme d'accueil après un transfert qui mette l'accent sur le suicide et qui soit fondé sur le transfert et l'échange d'informations, écrites (dossier médical du détenu, etc.) et verbales (échanges téléphoniques, etc.), avec les différents membres du personnel.

La deuxième forme d'action discutée porte sur la transformation de l'action des professionnels. Une fois le repérage effectué, ce qui peut être offert est une relation positive ou thérapeutique, dont sont espérés des effets préventifs. Les rapports constatent la pénurie de personnel psychologique et psychiatrique. Ils mettent également l'accent sur la nécessité d'associer l'ensemble des membres du personnel aux actions préventives et de définir le rôle de chacun. Un rapport insiste sur le fait que le milieu carcéral ne permet pas de transposer un «modèle médical», raison pour laquelle, «dans un pénitencier, les agents de sécurité, les agents de programme et les autres employés 
sont responsables, dans une large mesure, du dépistage et de la prévention de ces problèmes» (SCC, 1981a). Pour cette raison, la pluridisciplinarité est posée d'emblée comme un axe de travail important. Ce registre d'action nécessite la formation du personnel pour le sensibiliser à la question du suicide. Il s'agit ainsi de lutter contre «les préjugés» du personnel, qui reproduisent une vision morale et fataliste du suicide carcéral (SCC, 1982).

Ce registre d'action se concrétise pour la première fois en 1983 sous la forme d'un programme de formation à destination des employés des services correctionnels de la région de l'Atlantique. Ce programme centré sur le développement des attitudes, des connaissances et des aptitudes à détecter les détenus à risque ainsi que sur leur prise en charge vise à former des candidats chargés, à leur tour, de former leurs collègues (SCC, 1991). Les employés du SCC doivent y apprendre à identifier, comprendre et changer leur propre perception du suicide et des individus à risque, de même qu'à intervenir selon un modèle plus préventif que réactif. Après une période d'essai de deux ans, le programme de formation en prévention du suicide reçoit une évaluation favorable. Cette évaluation, fondée sur les propos recueillis auprès de plus de 70 membres du personnel du SCC et de 12 personnes détenues, conclut que sans l'existence du programme, «le nombre de suicides aurait été plus élevé» (Crookall et McLean, 1986).

La troisième forme d'action porte sur des décisions pénitentiaires, en particulier en matière de classement et de sanctions disciplinaires. C'est le point qui soulève le plus de questions par rapport au fonctionnement habituel des prisons. En effet, les cellules d'isolement comme les unités spéciales de détention sont les lieux repérés comme suicidogènes, en même temps qu'ils constituent la ressource ordinaire de l'institution pour gérer les prisonniers qu'elle considère comme posant des problèmes. Pour éviter que le placement en isolement ne soit (trop) néfaste pour les détenus identifiés à risque, le rapport de 1981(a) demande l'aménagement de secteurs de cellules de repos qui seraient à la fois des lieux d'exercice de personnels formés, mais aussi des lieux de plus grande surveillance, au moyen de caméras. Le rapport de 1982 (p. 83) pose des questions semblables et propose des efforts pour améliorer le classement et éviter de soumettre inutilement les détenus à «un niveau de sécurité de pénitencier excessif, surtout, les jeunes», comme de revoir la politique de placement à l'isolement pour y rendre possible «le support moral nécessaire». 
Le recours à cette pratique soulève pourtant d'importantes questions en matière de respect des droits des personnes détenues. D'une part, car, d'après le manuel de renseignements à l'usage des détenus édité par le SCC, l'isolement des détenus identifiés à risque est considéré comme une mesure non punitive qui leur permet de conserver tous leurs droits, sauf celui de se joindre aux autres détenus (SCC, s.d.). Dans la réalité, le rapport de 1982 nous apprend que les «détenus très dépressifs quittent leur bloc cellulaire pour être mis à l'écart souvent dans une cellule anti-suicide où il n'y a aucun équipement sanitaire ni matelas. Ils reçoivent alors très peu de contact humain». D'autre part, car la surveillance complète à laquelle sont soumis les détenus les plus à risque peut porter atteinte à leur vie privée. À ce sujet, le solliciteur général, John MacBeth, interrogé par un journaliste du Globe and Mail en 1977, explique que le SCC «[is] looking for ways to keep them [the inmates] under constant surveillance without interfering with their rights of privacy» (Trying to stop suicides in jail, MacBeth says, 1977). La politique de prévention du suicide est donc tout de suite en tension entre des droits à respecter: le droit à la vie, mais aussi le droit à l'intimité, menacé par les mesures de surveillance préventives.

À la suite des nombreuses recommandations émises par les experts et dans un effort de normalisation et de clarification des procédures, la première directive du commissaire est créée en 1987. Elle énonce les responsabilités des membres du personnel et les procédures à mettre en place pour prévenir le suicide et empêcher les automutilations (SCC, 1987). La directive clarifie le rôle du directeur d'établissement, qui doit, dit-elle, veiller à ce que les membres du personnel travaillant en contact avec les détenus reçoivent la formation de prévention du suicide évoquée ci-dessus et à l'élaboration d'ordres permanents détaillant les procédures de prévention. Parmi celles-ci, les trois pistes d'action envisagées par les SCC au début des années 1980 sont reprises. Il est ainsi demandé au personnel de «déterminer quels sont les délinquants susceptibles de se mutiler et de les mettre en observation»; de «communiquer, comme il se doit, aux membres du personnel intéressés les renseignements recueillis sur les délinquants en question»; ou encore de «créer une équipe interdisciplinaire chargée de surveiller tous les délinquants suicidaires ou susceptibles de se mutiler » (SCC, 1987). Dès la fin des années 1980, la prévention du suicide en milieu carcéral s'articule donc autour des procédures d'identification, de communication, de suivi et de surveillance. 


\section{Conclusion}

Au terme de cette décennie d'attention portée à la mort en prison, le suicide carcéral est sans aucun doute devenu un problème public, mobilisant divers acteurs et déclenchant des controverses sur l'analyse de ses causes et sur les moyens de prévention adaptés. Cette problématisation du suicide carcéral s'inscrit dans les transformations qui touchent le suicide de manière générale. Ce qui change durant les années 1970 et 1980 n'est pas le suicide carcéral lui-même, mais la manière dont le phénomène est inscrit dans un cadre plus large: une intensification de la préoccupation pour le phénomène suicidaire, la recherche active de populations à risque et la mobilisation d'une configuration d'acteurs pluriels interpellés par la thématique carcérale. En effet, l'attention portée par le SCC au suicide carcéral coïncide avec les efforts mis en place par les décideurs et les chercheurs pour enquêter et, in fine, recadrer la problématique du suicide: d'objet de réprobation morale et pénale, le suicide est devenu un objet de santé publique. S'ensuit le développement d'outils de mesure permettant de cibler les populations à risque, parmi lesquelles la population carcérale fait son entrée. Le suicide carcéral est donc d'emblée problématisé sous le prisme de la gestion des risques qui se développe au sein du milieu pénal et correctionnel (Feeley et Simon, 1992). S'ensuivent également les premières initiatives en matière de prévention et de politique publique pour lutter contre le phénomène suicidaire dans la société libre et en prison, justifiées par une démonstration quantitative répétée de l'ampleur du phénomène: si le suicide inquiète Santé et Bien-être Canada en 1974, il inquiète également le SCC, qui se trouve devant un phénomène de sursuicidité important.

Ces initiatives coïncident également avec la mobilisation de groupes militants et de journalistes qui, chacun de leur côté, dénoncent le nonrespect des droits des personnes détenues. Leur argumentaire est le suivant: la prison forme selon eux une atteinte au droit à la vie et cause les cas de suicide. Le suicide carcéral devient donc un objet de revendication de l'amélioration des conditions de vie des détenus. Les arguments avancés sont dès lors proches d'une logique «stat-activiste» selon laquelle les statistiques constituent un levier d'émancipation pour les entrepreneurs de problèmes qui les mobilisent (Bruno, Didier et Prévieux, 2014): chaque année, la prison tue un nombre (trop) important de personnes détenues. 
De plus, la manière dont le suicide est appréhendé et géré par les autorités correctionnelles au début des années 1980 s'inscrit dans les transformations plus larges de l'institution carcérale.

D'abord, la manière dont les prisons sont interpellées comme institutions suicidogènes prend place dans la promotion des droits des prisonniers et des instances de contrôle du monde pénitentiaire. Des militants et des journalistes partent de cette question pour ouvrir la boîte noire carcérale, notamment par le décompte des suicides et des demandes d'explications sur des cas qui auraient peut-être pu être évités. Ce faisant, ils «exposent la prison au regard et au jugement de la société» et contribuent à sa mise à l'épreuve (Cliquennois et Chantraine, 2009). Une des façons de prévenir le suicide est de sommer les autorités correctionnelles à livrer des informations, de les soumettre à de nouvelles expertises extérieures et, au moins en partie, de les rendre responsables et susceptibles de devoir rendre des comptes sur leur (in)action par ces mêmes acteurs. La formalisation de procédures s'inscrit dans cette logique tout en mettant l'accent sur la marge de manœuvre relative de l'institution et la difficulté de la concrétisation de telles actions: si la volonté du SCC de protéger la vie des détenus est présente, elle entre en conflit avec plusieurs des droits qui leur sont conférés.

Ensuite, la politique de prévention du suicide fait partie des actions d'ouverture des prisons, en particulier à d'autres corps professionnels que les agents de surveillance. On l'a vu, elle se formule très vite autour de la nécessité d'ouvrir des espaces de dialogue bienveillant, avec des intervenants, psychologues ou psychiatres contre les formes de mortification du fonctionnement traditionnel des prisons. Mais ces actions de prévention forment en même temps un bon révélateur des limites de cette ouverture. En effet, le répertoire des actions possibles demeure soumis aux contraintes d'une institution pénale et disciplinaire. La politique de prévention du suicide repose sur le développement de «savoirs-risques» qui vise à identifier et à classer les détenus et réguler la détention (Chantraine, 2006). Elle s'appuie également sur des mesures telles que la «mise à l'écart non punitive» (SCC, 1982) et la mise sous surveillance des détenus repérés. En ce sens, les politiques de prévention du suicide entreprennent d'emblée une dialectique vouée à durer entre la mise à l'écart, pour les détenus suicidaires, de l'arsenal le plus violent et contraignant des sanctions disciplinaires, et la conception de mesures préventives qui s'inscrivent néanmoins dans les registres de la contention et de la surveillance. Notre analyse rejoint donc celle de Cliquennois et Chantraine (2009) dans les prisons françaises: 
L'empêchement de la mort en détention, concrétisé... par un effort de réduction des risques suicidaires, infléchit l'intensité de certaines logiques et sanctions disciplinaires qui ne s'appliquent plus du fait des tendances suicidaires du détenu. Mais en retour, ce dispositif accroît la surveillance individualisée des détenus, venant ainsi réactiver les techniques traditionnelles de régulation de la détention. (p. 77)

Lorsque l'individu représente une menace pour lui-même, s'installe alors un dilemme important entre liberté et restriction, facilement repérable en prison. Ce dilemme n'est certes pas propre à l'institution carcérale, et peut certainement être posé comme outil d'analyse, par exemple des mesures de contrainte dans les hôpitaux psychiatriques. Le cadre carcéral est cependant singulier comme cadre d'exécution d'une peine et d'imposition d'une souffrance, qui rend complexe, si ce n'est aporétique, la conception de mesures qui donnent envie de vivre plus qu'elles n'empêchent de se donner la mort. À côté de mesures à visée thérapeutique, dont la portée est limitée par les moyens disponibles, l'accent est donc mis sur l'empêchement de la concrétisation des idées suicidaires par des mesures qui voisinent de près celles que la prison utilise pour surveiller, empêcher les évasions et maintenir l'ordre.

\section{Références}

23 suicides, 80 tries in federal prison since Sept, 1, 1969. (1971, 16 février). The Globe and Mail, p. 8.

A jury speaks on suicide in the cells. (1974, 30 novembre). The Globe and Mail, p. 6.

À quand la fermeture de Parthenais? (1986, 6 décembre). La Presse.

Association internationale de prévention du suicide. (1979). Communications: $10^{e}$ congrès international sur la prévention du suicide et les interventions en cas de crise. Ottawa, Ontario.

Aujard, M.-F. (2007) La suicidologie, un outil de gestion du comportement. Recherches sociographiques, 48(3), 161-174.

Batt-Moilo, A. (2005). Les programmes de prévention du suicide dans différents pays occidentaux: préalables, formulations et perspectives. Dans A. BattMoillo et A. Jourdain (dir.), Le suicide et sa prévention: émergence du concept, actualité des programmes. Rennes, France: Éditions de l'École Nationale de la Santé Publique.

Becker, H. S. (1963). Outsiders: studies in the sociology of deviance. Londres, Royaume-Uni: Free Press of Glencoe.

Beigel, A. et Russell, H. E. (1972). Suicide attempts in jails: prognostic considerations. Psychiatric Services, 23(12), 361-363. 
Bérard, J. (2014). Genèse et structure des conflits politiques sur les droits des détenus dans la France contemporaine. Déviance et Société, 38(4), 449-468.

Bernheim, J. C. (1987). Les suicides en prison. Montréal, Québec: Éditions du méridien.

Brown, H. (1978). National prison justice day - August $10^{\text {th }}$. Odyssey Newsletter, $1(1), 3$.

Bruno, I., Didier, E. et Prévieu, J. (2014). Statactivisme: comment lutter avec des nombres. Paris, France: Zones.

Burtch, B. E. et Erikson, R. V. (1979). The silent system: an inquiry into prisoners who suicide and [an] annotated bibliography. Toronto, Ontario: University of Toronto.

Campéon, A. (2005). Du faire mourir ou laisser vivre: le suicide en question. Dans A. Batt-Moillo et A. Jourdain (dir.), Le suicide et sa prévention: émergence du concept, actualité des programmes. Rennes, France: Éditions de l'École Nationale de la Santé Publique.

Cellard, A., Chapdelaine, E. et Corriveau, P. (2013). «Des menottes sur des pansements» : la décriminalisation de la tentative de suicide dans les tribunaux du Québec entre 1892 et 1972. Revue canadienne droit et société, 28(10), 83-98.

Chantraine, G. (2000). La sociologie carcérale: approches et débats théoriques en France. Déviance et Société, 24(3), 297-318.

Chantraine, G. (2006). La prison post-disciplinaire. Déviance et Société, 30(3), 273-288.

Chauvenet, A, Rostaing, C. et Orlic, F. (2008). La violence carcérale en question. Paris, France: Presses universitaires de France.

Cliquennois, G. et Chantraine, G. (2009). Empêcher le suicide en prison: origines et pratiques. Sociétés contemporaines, 75(3), 59-79.

Comité de santé mentale du Québec. (1982). Avis sur la prévention du suicide. Québec, Québec: Gouvernement du Québec.

Corriveau, P., Cauchie, J.-F. et Perreault, I. (2014). Enjeux autour de la responsabilité du geste suicidaire en institution carcérale. Champ pénal/Penal field, 11.

Crookall, P. et McLean, T. (1986). Évaluation du programme de formation en prévention du suicide dans la région de l'Atlantique. Ottawa, Ontario: Services correctionnels du Canada, Gouvernement du Canada.

Feeley, M. M. et Simon, J. (1992). The new penology: Notes on the emerging strategy of corrections and its implications. Criminology, 30(4), 449-474.

Foucault, M. (1975). Surveiller et punir. Paris, France: Éditions Gallimard

Foucault, M. (1976). La volonté de savoir: Histoire de la sexualité (Tome 1). Paris, France: Éditions Gallimard.

Gaucher, R. (1991). Organizing inside: Prison Justice Day (August 10 ${ }^{\text {th }}$ ), a non-violent response to penal repression. Journal of Prisoners on Prisons, 3(1-2), 93-110.

Godineau, D. (2012). S'abréger les jours: le suicide en France au XVIII siècle. Paris, France: Armand Colin. 
Goffman, E. (1968). Asiles: études sur la condition sociale des malades mentaux et autres reclus. Paris, France: Éditions de Minuit.

Goyer is astounded by 19 prison suicides. (1971, 13 janvier). The Globe and Mail, p. 3.

Guard carried a knife to cut down persons trying to hang selves. (1974, 29 novembre). The Globe and Mail, p. 1-2.

Internal probe can't find reason for 6 suicides at prison this year. (1973, 9 août). The Globe and Mail, p. 8.

Jail was warned before suicide. (1979, 25 octobre). The Globe and Mail, p. 62.

Lascoumes, P. et Le Galès, P. (2012). Sociologie de l'action publique ( $2^{\mathrm{e}}$ éd.). Paris, France: Armand Colin.

Leenaars, A. A. (2000). Suicide prevention in Canada: A history of a community approach. Canadian Journal of Community Mental Health, 19(2), 57-73.

Man waited 6 weeks for mental tests before suicide. (1976, 26 février). The Globe and Mail, p. 5.

Mary, P., Bartholeyns, F. et Béghin, J. (2007). La prison en Belgique: de l'institution totale aux droits des détenus? Déviance et Société, 30(3), 389-404.

Neveu, E. (2015). Sociologie politique des problèmes publics. Paris, France: Armand Colin.

Nurse says rule kept oxygen from hanged inmate. (1974, 22 novembre). The Globe and Mail, p. 8.

Payre, R. (2009). Les institutionnalisations improbables. Une sociologie historique prospective des sciences du gouvernement. Dans F. Buton et N. Mariot (dir.), Pratiques et méthodes de la socio-histoire (p. 69-81). Paris, France: Presses universitaires de France.

Petit, J. G. (1990). Ces peines obscures. La prison pénale en France (1780-1875). Paris, France: Fayard

Rostaing, C. et Touraut, C. (2016). Exposition en prison et hiérarchie morale des publics empêchés. Culture et Musées, (26), 23-46.

Santé et Bien-Être social Canada. (1974). Nouvelle perspective de la santé des Canadiens, document de travail. Ottawa, Ontario: Gouvernement du Canada

Santé et Bien-Être social Canada. (1987). Le suicide au Canada: rapport du Groupe d'étude national sur le suicide. Ottawa, Ontario: Gouvernement du Canada.

Service correctionnel Canada. (1987). Prévention du suicide et des automutilations (Directive du commissaire numéro 843). Ottawa, Ontario: Gouvernement du Canada. Repéré à https://www.publicsafety.gc.ca/lbrr/archives/cd-87-0101-843.pdf

Service correctionnel du Canada. (1979a). Étude des cas de suicide, de tentatives de suicide et d'auto-mutilation. Ottawa, Ontario: Gouvernement du Canada.

Service correctionnel du Canada. (1979b). [Rubrique]. Let's Talk, 4(14).

Service correctionnel du Canada. (1979c). [Rubrique]. Let's Talk, 4(19).

Service correctionnel du Canada. (1981a). Analyse statistique des automutilations et des suicides dans les pénitenciers canadiens. Ottawa, Ontario: Gouvernement du Canada 
Service correctionnel du Canada. (1981b). Réunion fédérale-provinciale. Responsables des services correctionnels - Livre des participants. Ottawa, Ontario: Gouvernement du Canada.

Service correctionnel du Canada. (1982). Étude et recommandations sur les suicides dans les pénitenciers au Québec. Ottawa, Ontario: Gouvernement du Canada.

Service correctionnel du Canada. (1984). Rapport du groupe d'étude: les sept suicides dans la région de l'Atlantique. Ottawa, Ontario: Gouvernement du Canada.

Service correctionnel du Canada. (1991). Propositions relatives à une politique à adopter dans le domaine de la prévention du suicide. Ottawa, Ontario: Gouvernement du Canada.

Service correctionnel du Canada. (s.d). Droit des détenus. Manuel de renseignements à l'usage des détenus. Ottawa, Ontario: Gouvernement du Canada.

Smith, T. (1980). Odyssey newsletter policy. Odyssey Newsletter, 1(9), 1.

Suicide spurs call for better inmate mental care. (1988, 9 août). Toronto Star.

Suicides shake public's view of Winnipeg's jail. (1977, 6 août). The Globe and Mail, p. 8.

Sykes, G. M. (1958). The society of captives; a study of a maximum security prison. New York, NY: Atheneum.

Topp, D. O. (1979). Suicide in prison. The British Journal of Psychiatry, 134(1), 24-27.

Trying to stop suicides in jail, MacBeth says. (1977, 29 octobre). The Globe and Mail, p. 5.

Vacheret, M. (2006). Gestion de la peine et maintien de l'ordre dans les institutions fédérales canadiennes: contrôle, pouvoir et domination: les «réussites» de la prison. Déviance et Société, 30(3), 289-304.

White, J. (2003). Recherche sur le suicide au Canada: Aperçu descriptif. Montréal, Québec: CRISE.

\section{Preventing prison suicide in Canada. The first steps of a public policy (1970-1987)}

ABSTRACT - This paper analyses how prison suicide became a public issue in the late 1970s and early 1980s and discusses the expertise and kinds of actions that originated from the Correctional Service of Canada. The origin of these reforms is shown to be the wider context of prison transformations, such as the development of inmates' rights, the opening of prisons to outside experts, particularly doctors, and the restructuring of the disciplinary function of the institution. The strongest suicide prevention measures, while only sketched out in the early 1980s, were already clearly focused on issues such as collecting and sharing information and classifying, assigning, and supervising suicidal inmates.

KEYWORDS - Suicide prevention, risk management, prison, public problems, Correctional service of Canada. 


\section{Prevenir el suicidio en prisión en Canadá. Los primeros pasos de una política pública (1970-1987)}

RESUMEN - El artículo analiza la constitución del suicidio carcelario como un problema público al final de los años 1970 y al comienzo de los años 1980, y rinde cuentas de las experiencias movilizadas y de los registros de acción esbozados por el Servicio Correccional de Canadá. Ubica el origen de estas reformas dentro del marco más amplio de las transformaciones del universo carcelario, en la intersección de la promoción de los derechos de los prisioneros, con el crecimiento del control de las prácticas penitenciarias, con la apertura creciente de las cárceles a profesionales externos, sobre todo a los médicos, y con la reconfiguración del funcionamiento disciplinario de la institución. El artículo muestra que a pesar de que no fueron esbozados sino hasta los años 1980, las líneas de fuerza y los puntos de tensión de las políticas de prevención del suicidio en prisión aparecen claramente, y según modalidades que serán luego perseguidas y profundizadas, sobre todo alrededor de los problemas de recolección y de intercambio de información, de clasificación, de afectación y de vigilancia de los detenidos considerados suicidas.

palabras clave - Prevención del suicidio, gestión de riesgos, prisión, problema público, servicios correccionales de Canadá. 